\title{
Simulating Male Selfish Strategy in Reproduction Dispute
}

\author{
P.M.C. de Oliveira and S. Moss de Oliveira.
}

Laboratoire PMMH, Ecole Supérieure de Physique et Chimie Industrielle, 10 rue Vauquelin, F-75231 Paris, France.

Visiting from Instituto de Física, Universidade Federal Fluminense; Av. Litorânea s/n, Boa Viagem, Niterói 24210-340, RJ, Brazil; pmco@if.uff.br, suzana@if.uff.br.

\begin{abstract}
We introduce into the Penna Model for biological ageing one of the possible male mechanisms used to maximize the ability of their sperm to compete with sperm from other males. Such a selfish mechanism increases the male reproduction success but may decrease the survival probability of the whole female population, depending on how it acts. We also find a dynamic phase transition induced by the existence of an absorbing state where no selfish males survive.
\end{abstract}

Keywords: Evolution, Ageing, Monte Carlo Simulation, Absorbing state. PACS: $87.10+\mathrm{e}, 87.23$-a, 05.65 -b.

\section{Introduction}

According to Promislow and Pletcher [1] during the last 20 years there have been incredible advances in our undestanding of the evolution of development, in molecular evolution, and so on. However, theories of ageing have remained relatively restricted to the mutation accumulation theory of Medawar [2, 3] or to the antagonistic pleiotropy theory of Williams [4]. In their opinion, there are major evolutionary questions that could play a very important role in the evolution of senescence. One of these questions concerns the conflict between sexes.

In flies and many other species females mate several times in a short period, and store the sperm of different males. Thus, males have evolved mechanisms to maximize the ability of their sperm to compete with those of other males, at the expenses of the female's fitness. For instance, one protein found in the fruit fly male ejaculate has been shown to be similar to a spider neurotoxin [5]. 
Here we introduce into the Penna model for biological ageing [7], which is based on the mutation accumulation theory, such a kind of male strategy, in order to study the consequences on population survival.

\section{The Penna model}

In the asexual version of the Penna model (see 6] for several applications) the genome of each individual is represented by a computer word of 32 bits. Each bit corresponds to one "year" in the individual lifetime, and consequently each individual can live at most for 32 "years". A bit set to one means that the individual will suffer from the effects of a deleterious inherited mutation (genetic disease) in that and all following years. One step of the simulation corresponds to reading one bit of all genomes. Whenever a new bit of a given genome is read, we increase by one the individual's age. The rules for the individual to stay alive are: 1) The number of inherited diseases (bits set to 1) already accumulated until its current age must be lower than a threshold $T$, the same for the whole population. 2) There is a competition for space and food given by the logistic Verhulst factor $V=1-N(t) / N_{\max }$, where $N_{\max }$ is the maximum population size the environment can support and $N(t)$ is the current population size. We will consider $N_{\max }$ five times larger than the initial population $N(0)$. At each time step and for each individual a random number between zero and one is generated and compared with $V$ : if it is greater than $V$, the individual dies independently of its age or genome.

If the individual survives until a minimum reproduction age $R$, it generates $b$ offspring in that and all following years. The offspring genome is a copy of the parent's one, except for some randomly chosen deleterious mutations introduced at birth. That is, if a bit 1 is randomly tossed in the parent's genome, it remains 1 in the offspring genome; however, if a bit zero is randomly tossed, it is set to 1 in the mutated offspring genome. In this way, for the asexual reproduction the offspring is always as good as or worse than the parent.

The sexual version of the Penna model was first introduced by Bernardes [8], followed by Stauffer et al. [9], who adopted a slightly different strategy. We are going to describe and use the second one. Now individuals are diploids, with their genomes represented by two bit-strings that are read in parallel. In order to count the accumulated number of mutations and compare it with the threshold $T$, it is necessary to distinguish between recessive 
and dominant mutations. A mutation is counted if two bits set to 1 appear at the same position in both bit-strings (inherited from both parents) or if it appears in only one of the bit-strings but at a dominant position (locus). The dominant positions are randomly chosen at the beginning of the simulation and are the same for all individuals.

The population is now divided into males and females. After reaching the minimum reproduction age $R$, a female randomly chooses a male with age also equal to or greater than $R$ to mate. To construct one offspring genome first the two bit-strings of the mother are cut in a random position (crossing), producing four bit-string pieces. Two complementary pieces are chosen to form the female gamete (recombination). Finally, $m_{f}$ deleterious mutations are randomly introduced. The same process occurs with the male's genome, producing the male gamete with $m_{m}$ deleterious mutations. These two resulting bit-strings form the offspring genome. The sex of the baby is randomly chosen, each with probability 50\%. This whole strategy is repeated $b$ times to produce the $b$ offspring. The Verhulst killing factor already mentioned works in the same way as in the asexual reproduction.

\section{Male selfish strategy and results}

To simulate the male ability to increase its own reproductive success we attribute to half of the male population a label, to play the role of a selfish gene. The offspring of a selfish male may inherit this gene with a given probability $p_{s}$. Non-selfish males produce only non-selfish offspring. The selfish male has the advantage that whenever it mates, the female generates $b+1$ offspring (instead of $b$ as the non-selfish males). On the other hand, the female pays the bill for this male advantage (as usual). We tested two different prices to be paid:

case 1) If a female mates with a selfish male she loses forever part of her energy to dispute for food and space; That is, her probability to die due to the Verhulst factor increases 20\% (or equivalently, for her the carrying capacity $N_{\max }$ becomes $20 \%$ smaller). Such a punishement occurs only once; if she mates again with another selfish male, nothing new happens.

case 2) Whenever a female mates with a selfish male, her genetic death age (programmed since birth as the age where the $T$-th genetic disease would appear) is decreased by one, i.e. the female dies one year before the expected date. Note that in this case there is always a price to be paid, that does not 
depend on the value of any random number, contrary to the previous one.

For both cases we observe that if the selfish gene inheritance probability $p_{s}$ is below a given value $p_{c}$, after many generations no selfish male remains in the population. When $p_{s}$ increases above $p_{c}$, the percentage of selfish males also increases, reaching $100 \%$ for $p_{s}=1$. In figure 1 we show how this percentage increases with increasing $p_{s}$, for case 1). Squares correspond to a larger population. The curve for case 2 ) is analogous. There is a dynamical phase transition between an absorbing state in which selfish males are extinct, and a state where they do survive. The order parameter of this transition is the fraction shown in figure 1.

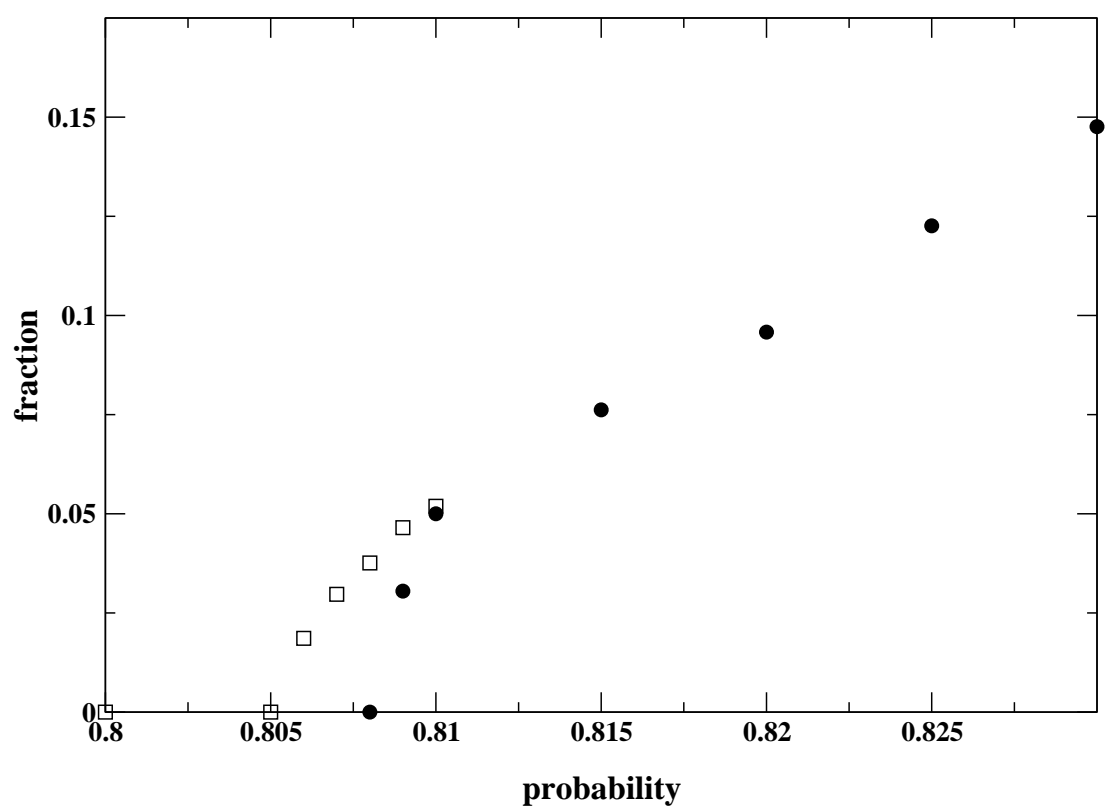

Figure 1: Final fraction of selfish males as a function of the inheritance probability $p_{s}$ for case 1.) Common parameters: threshold number of bad mutations $T=3$; minimum reproduction age $R=8$; birth rate $b=4$; mutation rates $m_{m}=m_{f}=1$; results averaged over the last 10,000 timesteps. Circles: initial population $P(0)=100,000$ and total number of timestpes $t=100,000$; Squares: $P(0)=200,000$ and $t=200,000$. 


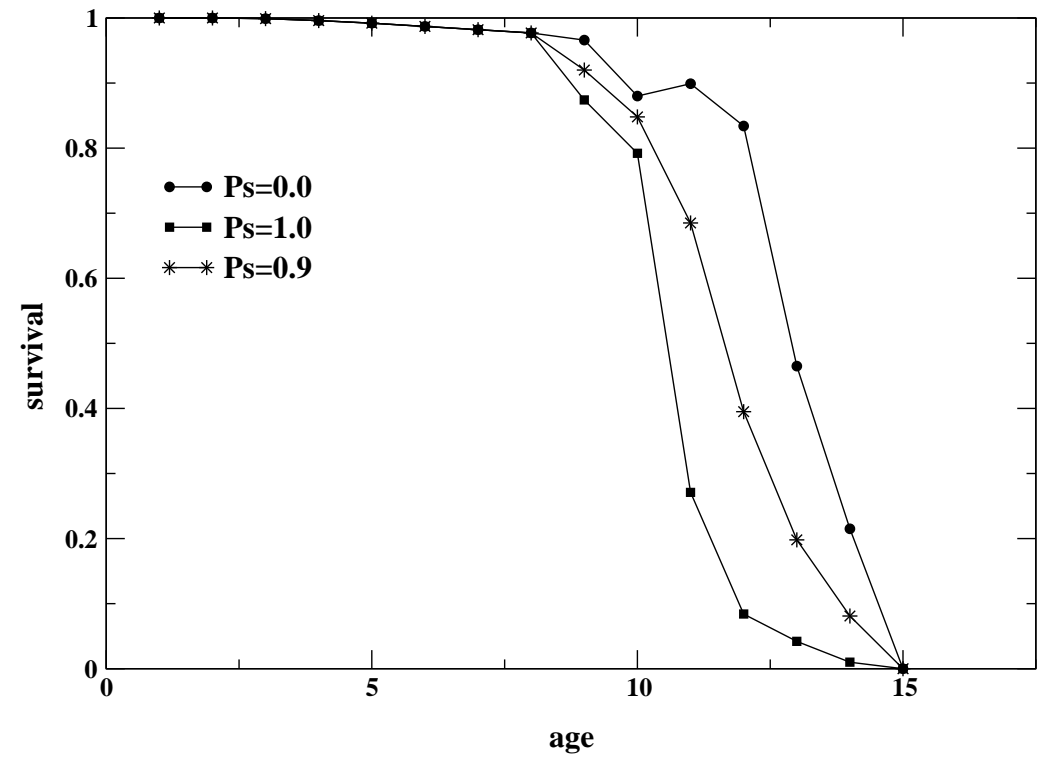

Figure 2: Female survival probabilities as a function of age for case 2). The parameters are the same as those of the larger populations of fig. 1. For any $p_{s}<p_{c}$, male and female survival probabilities are the same.

In both cases the total (already stable) population fluctuates around half of the value of the initial population, independently of the $p_{s}$ value. In the first case the male and female population sizes are always the same, as well as their survival probabilities as a function of age. (The survival probability $S(a)$ is defined as the ratio between the number of individuals with age $a+1$ and the number of individuals with age $a$.)

However, in the second case the female survival probability decreases with increasing values of $p_{s}$, in comparison to the male survival probability (which does not change at all). Results are shown in figure 2. Also the female population sizes are slightly smaller than the male ones, when $p_{s}>p_{c}$. For an initial population of 200,000 individuals (half of each sex), when $p_{s}<p_{c}$ both the male and female populations stabilize around 87,000. When $p_{s}=1$, the male population stabilizes around 88,000 individuals and the female one, around 84,000. 


\section{Conclusions}

We introduce into the Penna model for biological ageing a male strategy that improves its sperm ability to compete with sperm from other males. We obtain that depending on how this strategy works, it may decrease the female survival probability as a function of age. Considering as an order parameter the percentage of males carrying such an ability, we observe a phase transition between populations where no such males survive (after many generations) and populations where they succeed, depending on the probability of their offspring to inherit this "selfish gene".

Acknowledgements: To PMMH at ESPCI for the warm hospitality, to Sorin Tănase-Nicola for helping us with the computer facilities and to the Brazilian agency FAPERJ for financial support.

\section{References}

[1] D.E.L. Promislow and S.D. Pletcher, Mechanisms of Ageing and Development 123, 841 (2002).

[2] P.B. Medawar, Modern. Quart. 2, 30 (1946).

[3] P.B. Medawar, Un unsolved problem in Biology, H.K. Lewis, London (1952).

[4] G.C. Williams, Evolution 11, 398 (1957).

[5] M.F. Wolfiner, H.A. Harada, M.J. Bertram, T.J. Stelick, K.W. Kraus, J.M. Kalb, Y.O. Lung, D.M. Neubaum, M. Park and U. Tram, Insect Biochem. Mol. Biol. 27, 825 (1997).

[6] S. Moss de Oliveira, P.M.C. de Oliveira and D. Stauffer: Evolution, Money, War and Computers, Teubner, Stuttgart and Leipzig (1999).

[7] T.J.P. Penna, J. Stat. Phys. 78, 1629 (1995).

[8] A.T. Bernardes, J. Physique I 5, 1501 (1995). 
[9] D. Stauffer, P.M.C. de Oliveira, S. Moss de Oliveira and R.M. Zorzenon dos Santos, Physica A 231, 504 (1996); S. Moss de Oliveira, P.M.C. de Oliveira and D. Stauffer, Braz. J. Phys. 26, 626 (1996). 